\title{
Signal Reflection in Shortwave Multi-hop Transmission Mode
}

\author{
Xuehui Cui ${ }^{1, ~ a}$, Wei Xiang, b, * \\ ${ }^{1}$ College of Electrical and information Engineering, Southwest MinZu University of China, \\ Chengdu, Sichuan, 610225, P.R. China \\ a1466304633@qq.com,b3730544@qq.com \\ *Corresponding author
}

Keywords: Maritime communication, shortwave, ionosphere, reflection intensity, free space.

Abstract: For the purpose of reducing loss in signal propagation, this paper calculates numbers of hops on the surface of ground and sea and proposes methods for minimizing the loss of the signal and remain the ship in communication using the same multi-hop path in the process of multi-hop propagation mode of high-frequency signals. Four mathematical models are proposed to determine the loss of the signal in the process of propagation, parallel reflection model, calm ocean surface reflection model, turbulent ocean sphere reflection model and ground reflection model. The influential factors of signal is divided into noise and attenuation. Furthermore, the noise is divided into three categories, atmospheric radio noise, artificial radio noise, and cosmic noise. Attenuation is divided into free space propagation loss, ionospheric absorption loss, reflection loss, and extra system loss. This paper studies the shipboard receiver moving on a turbulent ocean range of the receiver on its received signal and defines the maximum communication time and communication area so as to calculate the holding time of the multi-hop path. It can be found that the value of the change of emission point incident angle per second on average. Thus, it can be ensured that the ship is always in communication with the transmitting station during the traveling process.

\section{Introduction}

Since the invention of Radio Communication technology, high-frequency communications have become the main role of long-range wireless communications ${ }^{[2]}$. At present, the communication technologies used by ships at sea mainly include single sideband shortwave radio and satellite mobile communication network. The modernization of offshore operations requires more information service capabilities ${ }^{[2]}$. Nevertheless, there is an un inevitable loss during signal propagation. Hence, we synthesize influencing factors, intending to develop a series of mathematical models to accommodate different circumstances through analyze of numbers of hops. 


\section{Models}

\subsection{Model I: Parallel Reflection Model}

Attenuation (loss)

In shortwave wireless transmission, ionospheric propagation loss can be expressed as follows:

$$
L_{b}=L_{s}+L_{i}+L_{g}+Y_{p}
$$

- Free space propagation loss $L_{s}$

Free space propagation loss is the decibel of the ratio of the radiated power to the power received by the lossless receiving antenna, we can get:

$$
L_{s}=10 \lg \frac{P_{T}}{P_{R}}
$$

Where, $P_{T}$ is antenna radiation power; $P_{R}$ is antenna receiving power of antenna $r$ away from emitter. Then the power density at the slope distance $r=\frac{P_{T}}{4 \pi r^{2}}$. The total area for receiving isotropic antennas in free space is $\lambda^{2} / 4 \pi$. So we get $P_{R}=\frac{P_{T}}{4 \pi r^{2}} \times \frac{\lambda^{2}}{4 \pi}$.

$$
L_{s}=32.44+20 \lg f+20 \lg r
$$

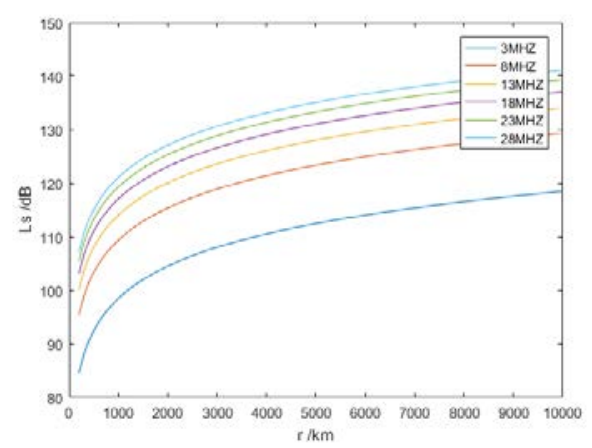

Fig. 1: The relationship between $\mathrm{r}$ and loss at different frequencies

Single-hop mode diagram is as follows:

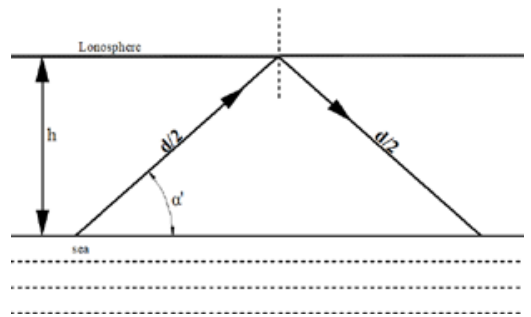

Fig. 2: Single-hop mode

On the basis of our inquiry, h is equal to $300 \mathrm{~km}$. We obtain:

$$
L_{s}=32.44+20 \lg \frac{2 n h f}{\sin \alpha^{\prime}}
$$


- Ionospheric absorption loss $L_{i}$

Three kinds of situations may occur when shortwave reaches the ionosphere: entering outer space through the ionosphere, being completely absorbed by the ionosphere or being reflected back to the Earth. However, the absorption is complicated by the random variation of ionosphere itself, so we use the semi-empirical formula in calculation.

$$
L_{i}=n I \frac{677.2 \sec \alpha_{100}}{\left(f+f_{H}\right)^{1.98}+10.2}
$$

Where, $f_{H}$ is magnetic rotation frequency at an altitude of $100 \mathrm{~km}$, the value of which is $1.4 \mathrm{MHz}$; $\alpha_{100}$ is incident angle at the height of $100 \mathrm{~km}$.

$$
\theta=\operatorname{arctg}\left[\left(\cos \frac{D}{2 a_{0}}-\frac{a_{0}}{a_{0}+110}\right) \sin \left(\frac{D}{2 a_{0}}\right)\right]
$$

We find out following parameters in the winter in Beijing, where, $I$ is absorption coefficient.

The value of which is $\left(1+0.0037 \bar{R}_{12}\right)(\cos 0.881 \chi)^{1.3} ; \bar{R}_{12}$ is average change of sunspot during 12 months and the value is 0.506 ; $\chi$ is the sun zenith angle. $\mathrm{D}$ is the arc distance from the transmitter to the receiver equal to $\frac{2 h}{\tan \alpha^{\prime}}$. Finally, we get $L_{i}=8.5008 n \mathrm{~dB}$. The range of incidence angle can be determined by following equation:

$$
\sin \alpha=\sqrt{1-\frac{80.8 N}{f^{2}}}
$$

Taking the ionospheric structure changes and long-term stable reception into account, we do not use MUF, but use FOT, which is known as the best working frequency while determining the line frequency. From Recommendation ITU-R M.1795, the FOT at 2: 00 p.m. during the day is $20 \mathrm{MHz}$.Finally, we have $\alpha_{\min }=\beta_{\min }=\arcsin \sqrt{1-\frac{80.8 \times 2 \times 10^{12}}{\left(20 \times 10^{6}\right)^{2}}}=50.5347^{\circ}$.

- Multi-hop reflection loss $\mathrm{L}_{g}$

According to our assumption that radio energy is distributed in horizontal and vertical polarization fields, the formula of multi-hop reflection loss $L_{g}$ can be deduced as follows:

$$
L_{g}=10 \lg \frac{\left|R_{v}\right|^{2}+\left|R_{h}\right|^{2}}{2}
$$

According to Snell law, the relative permittivity and conductivity of seawater are obtained.

$$
\begin{gathered}
\varepsilon_{r}=\left\{\begin{array}{l}
70, f \leq 2253.5895 \\
\frac{1}{a+b f+c f^{2}+d f^{3}+e f^{4}}, f>2253.5895
\end{array}\right. \\
\sigma=\left\{\begin{array}{l}
5.0, f \leq 1106.207 \\
\frac{r+s f+t f^{2}}{1+u f+v f^{2}+\omega f^{3}}, f>1106.207
\end{array}\right.
\end{gathered}
$$

Because of the frequency range we studied is 3 to $30 \mathrm{MHz}$, we deem $\varepsilon_{r}=70, \sigma=5.0$ here. Thus, 
$\varepsilon_{r}{ }^{\prime}=70-4500 j$. So we get the relationship between $\alpha^{\prime}$ and $L_{g}$ below:

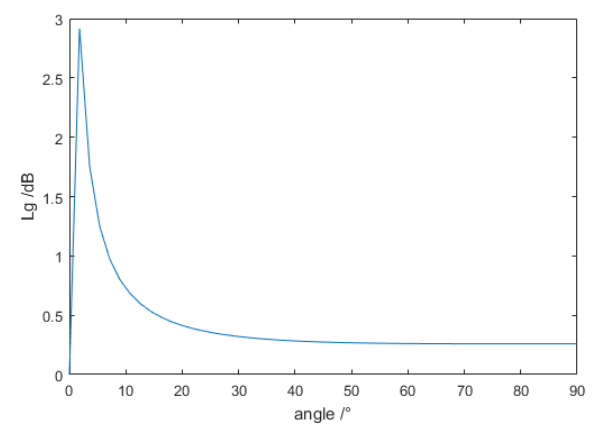

Fig. 3: The relationship between $\alpha^{\prime}$ and $L_{g}$

To get the maximum incidence angle and minimize the multi-hop reflection loss, we take $\alpha^{\prime}=20^{\circ}$. Thus, we get $\alpha_{\max }=70^{\circ}$. When the grazing incidence angle of 20, we get $L_{g}=0.414 \mathrm{~dB}$.

- Additional system loss $\mathrm{L}_{g}$

The extra system loss $\mathrm{L}_{\mathrm{p}}$ can simply be regarded as $14 \mathrm{~dB}$ according to Recommendation ITUR 339. We get:

$$
L_{b}=32.44+20 \lg f+20 \lg \frac{2 n h}{\sin \alpha^{\prime}}+8.5008 n+10 \lg \frac{\left|R_{v}\right|^{2}+\left|R_{h}^{2}\right|}{2}+14
$$

$>$ Noise

- Atmospheric radio noise

Atmospheric radio noise intensity is calculated as:

$$
E_{1}=F_{a}+10 \lg B+20 \lg f-96.8 \text {. }
$$

Where $B$ is effective noise bandwidth of receiver, here we take $B=3 \times 10^{6} \mathrm{~Hz}$.

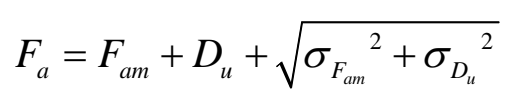

Where, $D_{u}$ is the upper tenth of the atmospheric radio noise figure $F_{a}$ in the time zone; $\sigma_{F_{a m}}$ is the standard deviation of $F_{a m} ; \sigma_{\mathrm{D}_{u}}$ is the standard deviation of $D_{u}$. The result is presented in the table below:

Table 1: $F_{a m}$ at $2 . p m$ in summer is $78 \mathrm{~dB}$ at $1 \mathrm{MHz}$

\begin{tabular}{|ccccccc|}
\hline frequency & $7.5 \mathrm{MHz}$ & $10 \mathrm{MHz}$ & $12.5 \mathrm{MHz}$ & $15 \mathrm{MHz}$ & $17.5 \mathrm{MHz}$ & $20 \mathrm{MHz}$ \\
\hline$F_{a m}$ & 51 & 45 & 37 & 32 & 27 & 21 \\
\hline$D_{u}$ & 9 & 7.5 & 6 & 5 & 4 & 3.2 \\
\hline$\sigma_{F_{a m}}$ & 3 & 3.2 & 3.5 & 3.6 & 3.9 & 4.2 \\
\hline$\sigma_{D_{u}}$ & 2.5 & 1.8 & 1.8 & 1.9 & 2 & 2.3 \\
\hline$F_{a}$ & 63.9051 & 55.6715 & 46.9357 & 41.0706 & 35.3829 & 28.9885 \\
\hline$E_{1}$ & 49.3776 & 43.6427 & 36.8451 & 32.5637 & 28.2149 & 22.9803 \\
\hline
\end{tabular}

We use MATLAB to achieve data fitting and gain the following equation: 


$$
E_{1}=-0.0024 f^{4}+0.1249 f^{3}-2.3580 f^{2}+16.4200 f+13.6143
$$

Based on above equation, we draw the fitting curve and compare it with real curve:

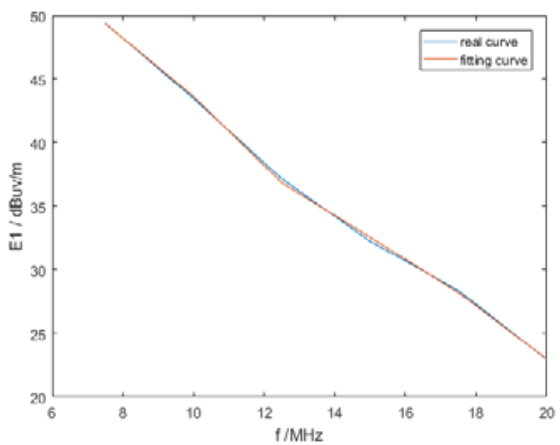

Fig. 4

We calculate that $s^{2}=0.0416$. The result obtained is in agreement with the experimental values.

- Artificial radio noise

Artificial radio noise mainly comes from various electric equipment and internal combustion engine ignition system. The equation of median of noise power is:

$$
E=c-d \lg f
$$

Table 2: The value of the parameter c, $\mathrm{d}$

\begin{tabular}{|ccc|}
\hline Environmental types & c & d \\
\hline Business district & 76.8 & 27.7 \\
\hline Residential area & 72.5 & 27.7 \\
\hline Countryside & 67.2 & 27.7 \\
\hline Quiet village & 53.6 & 28.6 \\
\hline Cosmic noise & 52.0 & 23.0 \\
\hline
\end{tabular}

The level of artificial radio noise is strong in urban industrial and commercial areas. The ocean is sparsely populated, and for the time being we think $E_{2}=0$.

- Cosmic noise

With the increase of frequency, the influence on the radio communication system above $1 \mathrm{GHz}$ is negligible. We get

$$
E_{3}=52-23 \lg f .
$$

According to Recommendation ITU-R P.533-12, we get the equation of field strength at any point of the shortwave sky wave is

$$
E=136.6+20 \lg f(M H z)+P_{t}(d B K W)+G_{t}(d B)-L_{b}(d B)
$$

Where, $P_{t} \mathrm{Pt}$ is the power of the transmitter; $G_{t}$ is the directional gain of the radioactive elevation angle of the transmitting antenna. The launch signal is $100 \mathrm{~W}$ high frequency constant carrier signal $G_{t}=1$.In the title, the transmitted signal is $100 \mathrm{~W}$ high frequency constant carrier signal. Thus, we get

$$
P_{t}(d B K W)=50 \mathrm{~dB}
$$

We refer to the definition of Signal-to-noise ratio and get $S N R=E-E_{1}-E_{2}-E_{3}$, solving the value of $n$ is 3 . 


\subsection{Model II: Calm Ocean Sphere Reflection Model}

We take the curvature of the earth, singled out mode into account, making the following graph.

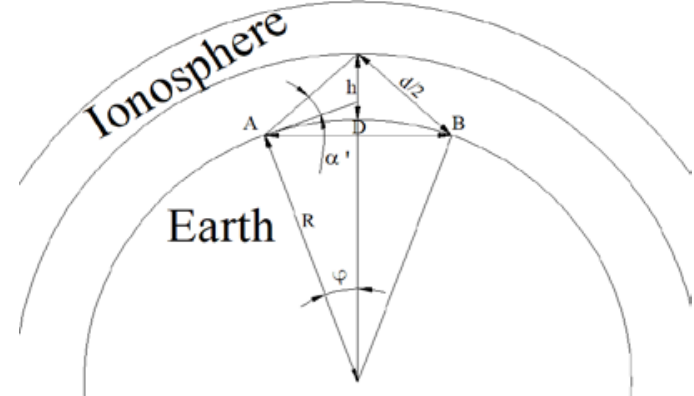

Fig. 4: Radio wave emission schematic

From the schematic above, we abstract the mathematical graph as follows:

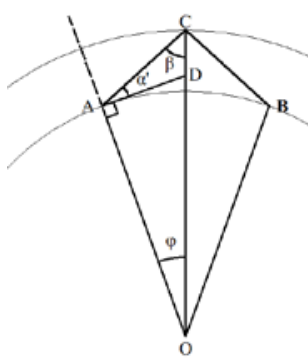

In the triangle AOC, we get

$$
r=2 \sqrt{(R+h)^{2}+R^{2}-2(R+h) R \cos \varphi}
$$

Finally, we obtain

$$
\alpha^{\prime}=\arcsin \frac{(R+h)^{2}-R^{2}-r^{2} / 4}{R r}
$$

When the curvature of the earth is considered, we have $\alpha \neq \beta$. But at this point, the minimum value of $\beta$ is invariant. That is, $\beta_{\min }=50^{\circ} 32^{\prime} 5^{\prime \prime}$. We use MATLAB to solve the range of the first reflection intensity is $79.672 \sim 80.0927 d B$. Meanwhile, we calculate $n=2$.

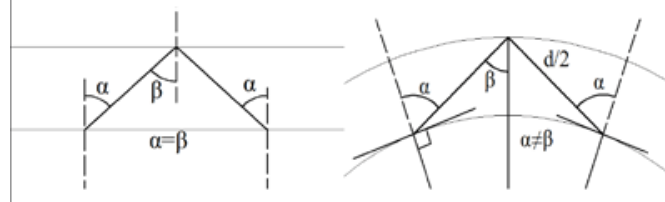

Fig. 5: Comparison of radio wave propagation in Model I and model II

\subsection{Model III: Turbulent Ocean Sphere Reflection Model}

For the problem of turbulent ocean, we use rough correction factor to approximate.

$$
\left\{\begin{array}{l}
R_{v}{ }^{\prime}=\rho R_{v} \\
R_{h}{ }^{\prime}=\rho R_{h}
\end{array}\right.
$$

According to our inquiry, we have: 


$$
\begin{gathered}
\rho=\frac{1}{\sqrt{3.2 g-2+\sqrt{(3.2 g)^{2}-7 g+9}}} \\
g=0.5\left(\frac{4 \pi h^{\prime} f \sin \alpha^{\prime}}{c}\right)^{2}
\end{gathered}
$$

Where, $h^{\prime}$ is root mean square deviation on sea surface, and we get $h^{\prime}=0.0051 w^{2}$ in view of Phillips wave model; $\mathrm{w}$ is wind speed near sea surface (unit is $\mathrm{m} / \mathrm{s}$ ).
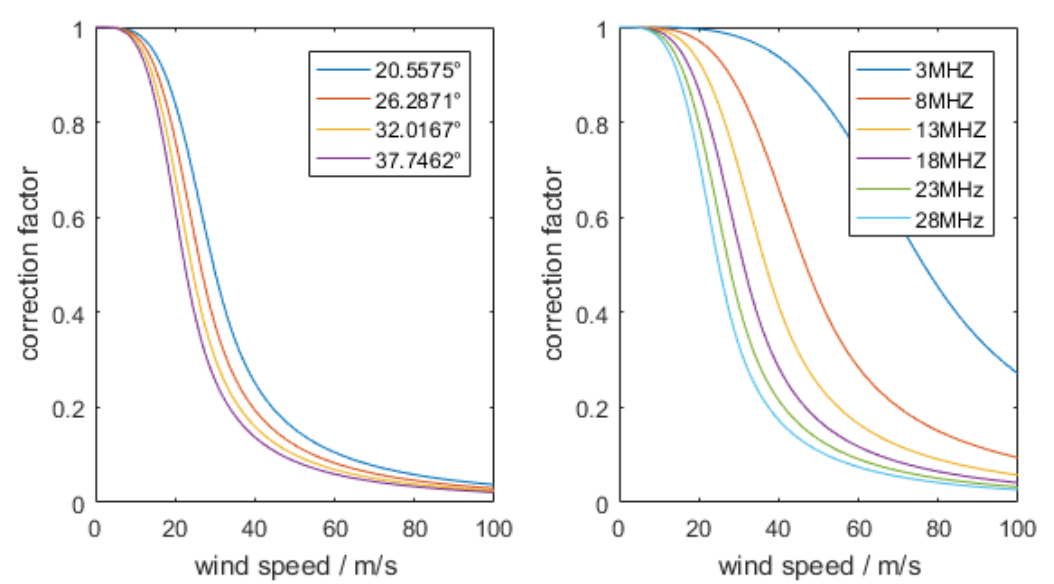

Fig. 6: Relationship between and the wind speed at different frequencies and different grazing incidence angles

Wind power level is usually in the $5 \sim 7$ level, so we take $w=36 \mathrm{~km} / \mathrm{h}=10 \mathrm{~m} / \mathrm{s}$. Thus, the first reflection intensity is $58.6564 \sim 58.699 \mathrm{~dB}$. The maximum of the numbers of hops is 1 . Compared with Calm Ocean, turbulent ocean loss increases 21.0156 21.3937dB .

\subsection{Model IV: Ground Reflection Model}

In this way, we divide the ground surface into flat ground and uneven surface. We take the mountain as an example to study the ground reflection so we draw the following diagrammatic sketch.

- Flat ground reflection

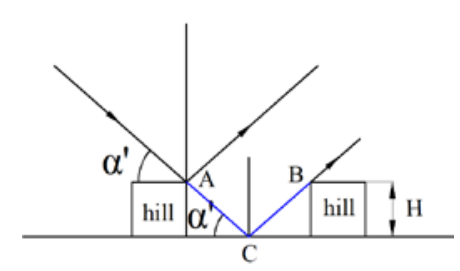

Fig. 7: Flat ground reflection

In slight of materials obtained, we have

$$
\begin{gathered}
\Delta r=\frac{\lambda}{4}=\frac{2 H_{\max }}{\sin \alpha^{\prime}} \\
\text { ground }=\left\{\begin{array}{l}
\text { flat, } h<H_{\max } \\
\text { uneven, } h>H_{\max }
\end{array}\right.
\end{gathered}
$$

Based on Calm Ocean Sphere Reflection Model, we change the dielectric constant and conductivity of sea to that on the ground. 
Table 3: Ground parameters

\begin{tabular}{ccccc|} 
Ground form & \multicolumn{2}{c}{ The scope of the changes } & \multicolumn{2}{c|}{ average value } \\
& $\varepsilon_{r}$ & $\sigma /(\Omega \bullet m)^{-1}$ & $\varepsilon_{r}$ & $\sigma /(\Omega \bullet m)^{-1}$ \\
\hline wet soil & $10 \sim 30$ & $3 \times 10^{-3} \sim 2.4 \times 10^{-2}$ & 10 & $10^{-2}$ \\
\hline dry ground & $3 \sim 4$ & $1.1 \times 10^{-5} \sim 2 \times 10^{-2}$ & 4 & $10^{-3}$ \\
\hline
\end{tabular}

We get the range of first reflection intensity is 67.29 67.33dB. Meanwhile, we calculate $n=2$.

- Uneven ground reflection

The mountain changes the ground incident angle of the radio waves. The diagram is as follows.

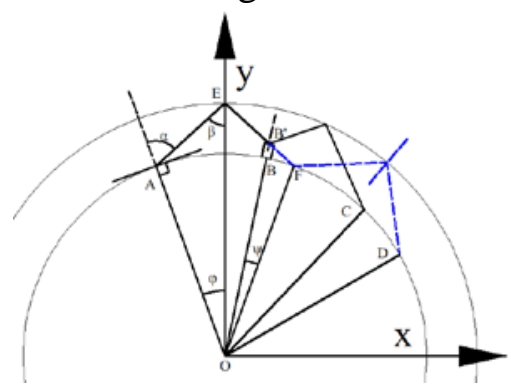

Fig. 8: Uneven ground reflection $(\mathrm{n}=2)$

Set up the axis as shown in the figure. We calculate the analytic formula of line OB and EF:

$$
\left\{\begin{array}{l}
y_{1}=x \tan \left(\frac{\pi}{2}-\varphi+\psi\right)(0<\psi<\varphi) \\
y_{2}=-\tan \left(\alpha^{\prime}+\varphi\right)(x-R \sin \varphi)+R \cos \varphi
\end{array}\right.
$$

Let $y_{1}=y_{2}$, we have

$$
\left\{\begin{aligned}
O B^{\prime} & =\sqrt{x^{2}+y^{2}} \\
H & =O B^{\prime}-R
\end{aligned}\right.
$$

We get the relationship between $H$ and $\psi$. To be closer to reality, we introduce $\overparen{A B}$ representing distance between mountain and emitter point.

$$
\overparen{A B}=R(\varphi+\psi)
$$

We use MATLAB to draw the following curves:

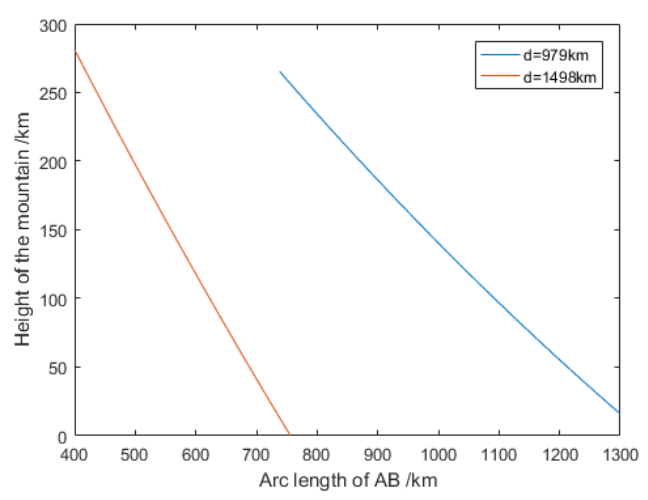

Fig. 9: Relationship between $\overparen{A B}$ and $\mathrm{H}$ 
According to the geometric relationship in above figure, we can list equations below:

$$
\left\{\begin{array}{l}
\Delta D=D_{2}-D_{1}+L_{1}+L_{2} \\
t_{\max }=\frac{\Delta D}{v} \\
\Delta \alpha=\alpha_{\max }-\alpha_{\min }
\end{array}\right.
$$

Here, we introduce the equation $\omega_{\alpha}=\frac{\Delta \alpha}{t_{\max }}$. Where, $\omega_{\alpha}$ refers to the change of emission point incident angle per second on average. We suppose that the speed of the vessel is. The length of antenna and ship is $39.41 \mathrm{~m}$.

$$
\begin{aligned}
\omega_{\alpha} & =2.52 \times 10^{-4}\left({ }^{\circ} / \mathrm{s}\right) \\
t_{\max } & =77132.155 \mathrm{~s}=21.4 \mathrm{~h}
\end{aligned} .
$$

We can draw the conclusion that we can ensure that the ship is always in communication during the traveling process through the adjustment of $\omega_{\alpha}$. At the same time, the ship can travel $21.4 h$ using the same multi-hop path.

\section{Conclusion}

This paper uses the number of hops to measure the loss of the signal in the process of propagation. We have analyzed the reflection state taking shortwave for example among radio waves. This paper compares ground reflection extent and calm ocean surface reflection and extends signal propagation from sea to ground. We find the value of the change of emission point incident angle per second on average. Thus, we can ensure that the ship is always in communication with the transmitting station during the traveling process.

\section{Acknowledgement}

This work was partially supported by Sichuan Youth Science and Technology Innovation Research Team (2017TD0028). Also was supported by the Fundamental Research Funds for Central University, Southwest Minzu University (2017NZYQN45).

\section{References}

[1] Davila C v. Comparison of HF Ground wave Propagation Models, NavalPS. 1993(1): l 14.

[2] Xu Biao, Wan Sha, Jiang Jianxing. Simulation and Measured of the Ionosphere Effect to Shortwave Sky Wave [J]. Digital Communication World, 2017, 3: 025. 